\title{
Triptolide reduces proliferation and enhances apoptosis of human non-small cell lung cancer cells through PTEN by targeting miR-21
}

\author{
XIAOFANG LI $^{1 *}$, AIMIN ZANG ${ }^{1 *}$, YOUCHAO JIA ${ }^{1}$, JINCHAO ZHANG $^{2}$, WUFANG FAN ${ }^{3}$, JIA FENG $^{1}$, \\ MINDAN DUAN $^{1}$, LEI ZHANG $^{1}$, RAN HUO $^{1}$, JIN JIAO $^{1}$ and XIAOWEI ZHU ${ }^{1}$ \\ ${ }^{1}$ Hebei Key Laboratory of Cancer Radiotherapy and Chemotherapy, Department of Medical Oncology, \\ Affiliated Hospital of Hebei University, Baoding, Hebei 071000; ${ }^{2}$ College of Chemistry and Environmental Science, \\ Chemical Biology Key Laboratory of Hebei Province; ${ }^{3}$ College of Life Sciences, \\ Hebei University, Baoding, Hebei 071002, P.R. China
}

Received March 21, 2015; Accepted December 1, 2015

DOI: $10.3892 / \mathrm{mmr} .2016 .4844$

\begin{abstract}
Triptolide is used in traditional Chinese medicine. It has the advantages of a unique mechanism of action, a wide antitumor spectrum, multiple targets, multi-channel effects and low toxicity. The current study was conducted to evaluate whether the potential anticancer effects of triptolide reduces proliferation and enhances apoptosis of human non-small cell lung cancer (NSCLC) cells, and to assess the underlying anticancer mechanisms. In PC-9 cells, treatment with triptolide reduced cell proliferation and increased cell apoptosis and caspase-3 and 9 activity. Triptolide treatment reduced miR-21 expression and enhanced phosphatase and tensin homolog (PTEN) protein expression levels in the PC-9 cells. Furthermore, the upregulation of miR-21 expression levels suppressed the effect of triptolide on cell viability and PTEN protein expression levels in PC-9 cells. To the best of our knowledge, the present study is the first to demonstrate that triptolide reduced the proliferation and enhanced the apoptosis of human NSCLC cells through PTEN by targeting miR-21.
\end{abstract}

\section{Introduction}

Lung carcinoma is one of the most common malignant tumors worldwide and the incidence nearly doubled in Asia over the

Correspondence to: Mr. Youchao Jia, Hebei Key Laboratory of Cancer Radiotherapy and Chemotherapy, Department of Medical Oncology, Affiliated Hospital of Hebei University, 212 Yuhua East Road, Baoding, Hebei 071000, P.R. China

E-mail: youchaojia@yeah.net

${ }^{*}$ Contributed equally

Key words: triptolide, non-small cell lung cancer, phosphatase and tensin homolog, miR-21 past ten years (1). The most common type of lung carcinoma is non-small cell lung cancer (NSCLC), which accounts for $80-85 \%$ of cases (2). In the past, due to the lack of effective treatment or early diagnosis, patient postoperative survival was poor, and tumor metastasis and recurrence determined the survival condition of patients (3).

MicroRNAs (miRNAs) are small non-coding RNA molecules, that are 21-25 nucleotides in length (4). They can be assembled into a ribonucleoprotein complex and are activated to target specific mRNA, by binding with the complementary sequence of the targeted gene. This results in the degradation or translational inhibition of the targeted mRNA molecule, to reduce the expression of target genes involved in cell proliferation, differentiation, apoptosis and death processes (5). Previous studies have observed that miRNAs are associated with the occurrence, development and prognosis of cancer, and can be used as molecular markers and guides in clinical diagnosis and treatment. miR-21 is one of the most important miR molecules out of $>700$ types of miRs that have been identified (6). Furthermore, previous studies have identified increased miR-21 expression levels in various types of cancer including liver (7), breast (8) and lung (9) cancer.

Phosphatase and tensin homolog (PTEN) is a newly identified tumor-suppressor gene. It exists in a variety of healthy tissues, however it can also be expressed in the tissues of malignant tumors (10). At present, it is clear that the association of multiple signaling pathway factors and PTEN serves an important role in cell growth, apoptosis and signal transduction, and participates in the occurrence and development of tumor malignancies (11).

Triptolide is isolated from the Tripterygium wilfordii plant. Components of Tripterygium wilfordii include highly activated diterpene lactone epoxide compounds, such as triptolide (12). It has numerous pharmacological purposes, including anti-inflammatory, immunosuppressive and anticancer activities (13). It is clinically used in the treatment of arthritis and autoimmune disorders, various types of cancer, organ transplant, kidney disease and asthma (14). In the present study, the antitumor activity of triptolide and the possible effects on 
PTEN and miR-21 expression were investigated, as its effect in human NSCLC cells remains unclear.

\section{Materials and methods}

Chemicals. RPMI-1640 medium and fetal bovine serum (FBS) was obtained from Thermo Fisher Scientific, Inc. (Waltham, MA, USA). 3-(4,5-dimethylthiazol-2-yl)-2,5-diphenyltetrazolium bromide (MTT) was obtained from Sigma-Aldrich (St. Louis, MO, USA). An annexin V-fluorescein isothiocyanate (FITC)/propidium iodide (PI) apoptosis assay kit was obtained from Nanjing KeyGen Biotech Co., Ltd. (Nanjing, China).

Cell lines and cell growth. The human lung cancer cell line PC-9 was provided by the Experimental Center of Hebei University (Baoding, China) and maintained in RPMI-1640 medium with $10 \% \mathrm{FBS}, 100 \mathrm{U} / \mathrm{ml}$ penicillin and $100 \mathrm{mg} / \mathrm{ml}$ streptomycin (both from Wuhan Procell Lite Science \& Technology Co., Ltd., Wuhan, China) in $37^{\circ} \mathrm{C}$ incubator with $5 \%$ carbon dioxide.

Cell viability. The in vitro effects of triptolide on cell viability of PC-9 cells was determined by the MTT assay. PC-9 cells were seeded at a density of $1 \times 10^{4}$ cells/well and plated onto a 96-well plate. Cells were exposed to different concentrations of triptolide $(0,10,25$ or $50 \mathrm{nM})$ for 3 days. Subsequently, $20 \mu 10.5 \%$ MTT solution with phosphate-buffered saline (Wuhan Procell Lite Science \& Technology Co., Ltd.) was added to each well and incubated for $4 \mathrm{~h}$ at $37^{\circ} \mathrm{C}$ with $5 \%$ carbon dioxide. Following the incubation period, the culture medium was replenished with fresh medium and $200 \mu 1$ dimethyl sulfoxide (Sigma-Aldrich) were added to each well and shaken for $20 \mathrm{~min}$ at room temperature (PZ300; Wuhan LEHD Ruihua Instruments Equipment Co., Ltd, Wuhan, China). The optical density of each well was measured at $492 \mathrm{~nm}$ using a Multiskan MS Plate Reader (LabSystems, Inc.; Thermo Fisher Scientific, Inc. USA).

Annexin V-FITC/PI apoptosis assay. The in vitro effect of triptolide (T3652; Sigma-Aldrich) on the cell viability of PC-9 cells was determined by the annexin V-FITC/PI assay. PC-9 cells were seeded at the density of $1 \times 10^{6}$ cells/well and plated onto a 6-well plate. Cells were exposed to different concentrations of triptolide $(0,10,25$ or $50 \mathrm{nM})$ for 2 days. Annexin V-FITC and PI were added to the cells and were incubated for $10 \mathrm{~min}$ at room temperature in the dark according to the manufacturer's instructions. Cell apoptosis was examined by flow cytometry (LSRII; BD Biosciences, San Jose, CA, USA).

Analysis of caspase-3 and 9 activation. PC-9 cells were seeded at a density of $1 \times 10^{6}$ cells/well and plated onto a 6 -well plate. Cells were exposed to different concentrations of triptolide $(0,10,25$ or $50 \mathrm{nM})$ for 2 days. PC-9 cells were prepared in cell lysis buffer (1067-100; Amyjet Scientific Inc., Wuhan, China) for $30 \mathrm{~min}$ on ice and then centrifuged at $12,000 \mathrm{x} \mathrm{g}$ for $10 \mathrm{~min}$ at $4^{\circ} \mathrm{C}$. The concentrations of the proteins of interest were determined using the Pierce BCA Protein Assay kit (BD Biosciences). Equal quantities of protein $(30 \mu \mathrm{g})$ were mixed with the caspase-9 substrate Ac-LEHD-pNA (E607115,
Sangon Biotech Co., Ltd., Shanghai, China) and the caspase-3 substrate Ac-DEVD-pNA (E607103-0200, Sangon Biotech Co., Ltd.)), and incubated at $37^{\circ} \mathrm{C}$ for $2 \mathrm{~h}$ in the dark. The optical density of each well was measured at $405 \mathrm{~nm}$ with a spectrophotometer.

Reverse transcription-quantitative-polymerase chain reaction (RT-qPCR). Total RNA in PC-9 cells was extracted with TRIzol reagent (Thermo Fisher Scientific, Inc.) and miRNAs were specifically amplified with TaqMan microRNA assays (Thermo Fisher Scientific, Inc.) according to the manufacturer's instructions. Quantification of the miRNAs was performed using SYBR-Green qPCR. The primers were used as follows: miR-21, F 5'-GGGGGTACCCTTCAGGAAGCT GGTTTC-3' and R 5'-GGGGATATCTACATGTGAGGCAG GTTCTCAC-3'; U6, F 5'-CGCTTCGGCACATATACTA-3' and R 5'-CGCTTCACGAATTTGCGTGTCA-3'.

Western blotting. PC-9 cells, treated with $25 \mathrm{nM}$ triptolide or miR-21 plasmids, were prepared in cell lysis buffer for $30 \mathrm{~min}$ on ice and then centrifuged at $12,000 \mathrm{x}$ g for $10 \mathrm{~min}$ at $4^{\circ} \mathrm{C}$. The protein concentrations of the samples were determined by the Pierce BCA protein assay kit according to the manufacturer's instructions. Equal quantities of protein $(30 \mu \mathrm{g})$ were loaded and fractionated by $12 \%$ SDS-PAGE gel (Wuhan Procell Lite Science \& Technology Co., Ltd.) at $80 \mathrm{~V}$ for $15 \mathrm{~min}$, and transferred to nitrocellulose membranes (Santa Cruz Biotechnology Inc., Dallas, TX, USA). Membranes were incubated with PTEN Antibody (F-1) (mouse monoclonal immunoglobulin $\mathrm{G}_{1}$; cat no., sc-393186; dilution, 1:1,500; Santa Cruz Biotechnology) and rabbit polyclonal $\beta$-actin (cat no., D110007; dilution, 1:500; Sangon Biotech Co., Ltd.) primary antibodies overnight at $4^{\circ} \mathrm{C}$. They were then incubated with the appropriate conjugated to a bovine anti-mouse immunoglobulin G-horseradish peroxidase-conjugated secondary antibodies (cat no., sc-2371; dilution, 1:2,000; Santa Cruz Biotechnology). Membranes were detected using electrochemiluminescence detection system (Bio-Rad Laboratories, Inc., Hercules, CA, USA) and observed using a luminescent image analyzer (Image Quant LAS4000 mini, GE Healthcare, Sweden).

Transfection. miR-21 (5'-AACAUCAGUCUGAUAAGC UAUU- $3^{\prime}$ ) and negative control plasmids (5'-CAGUACUUU UGUGUAGUACAA-3') were designed and purchased from Sangon Biotech Co., Ltd.. PC-9 cells were seeded at a density of $1 \times 10^{6}$ cells/well and plated onto a 6 -well plate for $24 \mathrm{~h}$. miR-21 $(100 \mathrm{ng} / \mathrm{ml})$ and negative control plasmids $(100 \mathrm{ng} / \mathrm{ml})$ were transiently transfected into PC-9 cells using Lipofectamine 2000 transfection reagent (Thermo Fisher Scientific, Inc.), according to the manufacturer's instructions. After $24 \mathrm{~h}$ of transfection, PC-9 cells were used to perform this experiment.

Statistical analysis. Data are presented as the mean \pm standard deviation and analyzed using SPSS software, version 17.0 (SPSS, Inc., Chicago, IL, USA). The statistical significance of results were determined using Student's t-test. $\mathrm{P}<0.05$ was considered to indicate a statistically significant difference. 


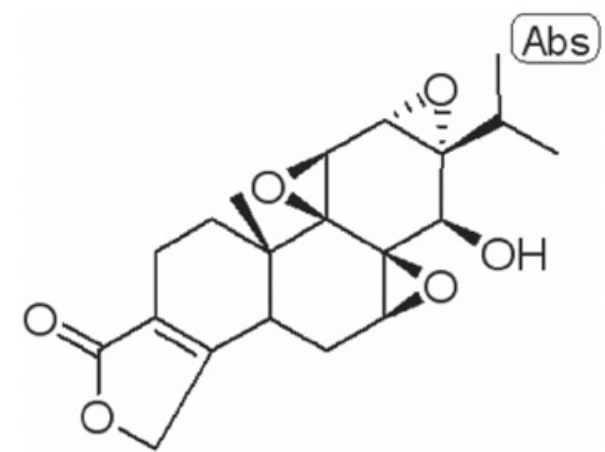

Figure 1. Chemical structure of triptolide.

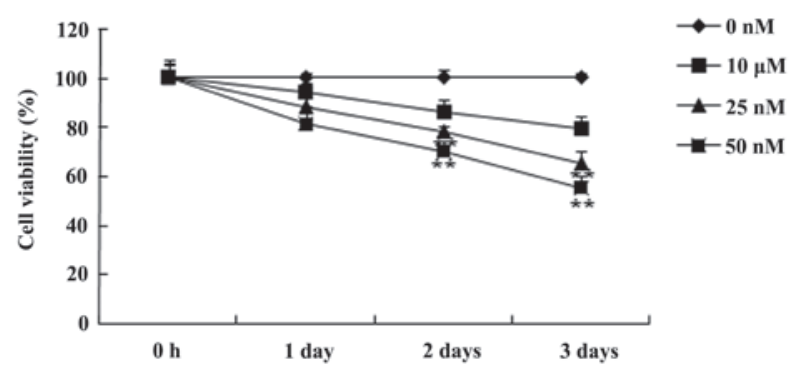

Figure 2. 3-(4,5-dimethylthiazol-2-yl)-2,5-diphenyltetrazolium bromide assay demonstrating the in vitro effect of triptolide on cell viability of PC-9 cells. ${ }^{* *} \mathrm{P}<0.01$ vs. $0 \mathrm{nM}$ triptolide-treated control group.

\section{Results}

Effect of triptolide on cell viability of PC-9 cells. The chemical structure of triptolide (98\% purity) is presented in Fig. 1. For the experimental purposes of the current study, triptolide was dissolved in physiological saline. The study investigated the predicted antitumor effect of triptolide on cell viability using the MTT assay. Fig. 2 demonstrates that the treatment with triptolide inhibited the cell viability of PC-9 cells in a timeand dose-dependent manner. The data additionally suggested that the cell viability of PC-9 cells was effectively inhibited by 25 or $50 \mathrm{nM}$ of triptolide after 2 or 3 days compared with the $0 \mathrm{nM}$ control group $(\mathrm{P}<0.01)$. These data suggested that triptolide may inhibit human NSCLC cell viability.

Effect of triptolide on cell apoptosis of PC-9 cells. To investigate the predicted antitumor effect of triptolide on cell apoptosis in human NSCLC, an annexin V-FITC/PI apoptosis assay was conducted. As demonstrated in Fig. 3, following a treatment period of 2 days, triptolide ( 25 or $50 \mathrm{nM}$ ) increased cell apoptosis compared with the control group $(\mathrm{P}<0.01)$. These data suggested that triptolide may promote human NSCLC cell apoptosis.

Effect of triptolide on caspase-3 and 9 activity in PC-9 cells. To further investigate the predicted antitumor effect of triptolide on cell apoptosis in human NSCLC, caspase-3 and 9 were measured in PC-9 cells. As demonstrated in Fig. 4, caspase-3 and 9 were effectually activated by triptolide treatment $(25$ or $50 \mathrm{nM})$ compared with the control group $(\mathrm{P}<0.01)$. These data suggested that triptolide may promote human NSCLC cell apoptosis by activation of the caspase- 3 and 9 pathway.

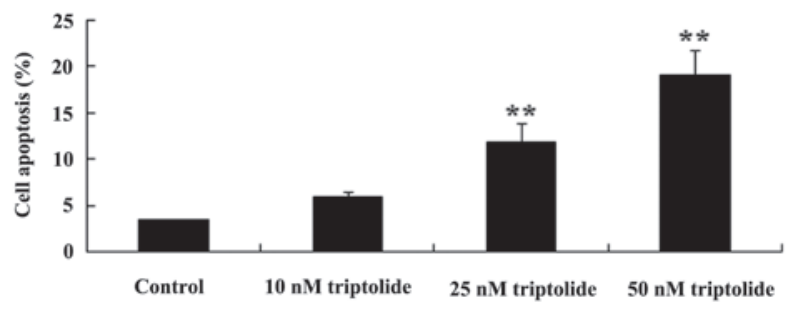

Figure 3. Annexin V-fluorescein isothiocyanate/propidium iodide assay demonstrating the in vitro effect of triptolide on cell apoptosis of PC-9 cells. ${ }^{* * *} \mathrm{P}<0.01$ vs. $0 \mathrm{nM}$ triptolide-treated control group.



Figure 4. In vitro effect of triptolide on (A) caspase-3 and (B) caspase-9 in PC-9 cells. ${ }^{* *} \mathrm{P}<0.01$ vs. $0 \mathrm{nM}$ triptolide-treated control group.



Figure 5. Reverse transcription-quantitative polymerase chain reaction assay demonstrating the in vitro effect of triptolide on miR-21 expression in PC-9 cells. ${ }^{* *} \mathrm{P}<0.01$ vs. $0 \mathrm{nM}$ triptolide-treated control group. miR-21, microRNA-21.

Effect of triptolide on miR-21 expression in PC-9 cells. RT-qPCR was utilized to assess the predicted antitumor effect of triptolide on miR-21 expression in PC-9 cells. As demonstrated in Fig. 5, the administration of triptolide (25 or $50 \mathrm{nM}$ ) suppressed miR-21 expression in PC-9 cells compared with the control group $(\mathrm{P}<0.01)$. These data suggested triptolide may weaken miR-21 expression in human NSCLC cells.

Effect of triptolide on PTEN protein expression in PC-9 cells. To identify the predicted antitumor effect of triptolide on PTEN protein expression in human NSCLC, PTEN protein expression levels were measured in PC-9 cells using western 
A

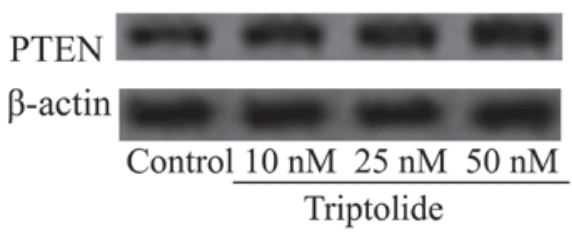

B

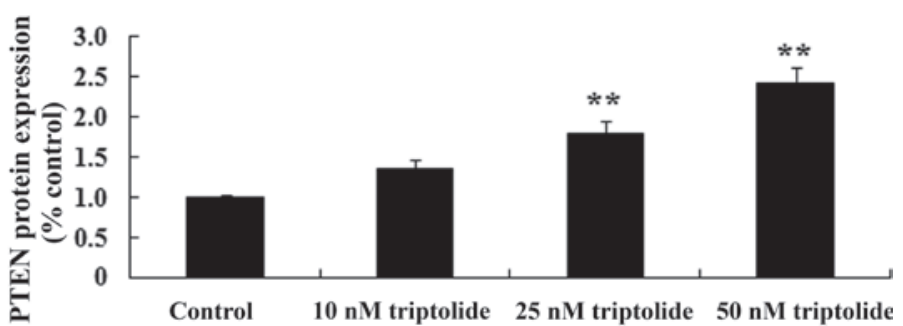

Figure 6. In vitro effect of triptolide on PTEN protein expression in PC-9 cells. (A) Western blot analysis and (B) statistical analysis of PTEN protein expression levels in vitro. ${ }^{* *} \mathrm{P}<0.01$ vs. $0 \mathrm{nM}$ triptolide-treated control group. PTEN, phosphatase and tensin homolog. PTEN, phosphatase and tensin homolog.


Figure 7. Upregulation of miR-21 influences the effect of triptolide treatment on cell viability in PC-9 cells. miR-21 plasmids influence (A) the miR-21 expression levels and (B) cell viability in PC-9 cells. ${ }^{* *} \mathrm{P}<0.01$ vs. $0 \mathrm{nM}$ triptolide-treated control group; ${ }^{\# \#} \mathrm{P}<0.01$ vs. 25 nM triptolide-treated group. miR-21, microRNA 21.

A

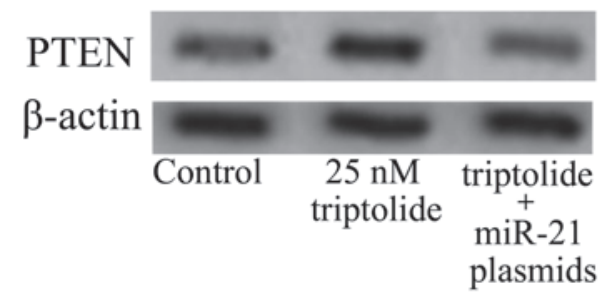

B

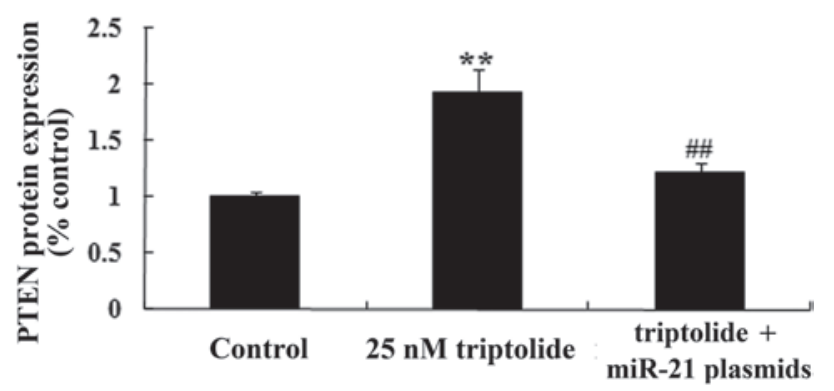

Figure 8. Upregulation of miR-21 influences the effect of triptolide on PTEN protein expression levels in PC-9 cells. (A) miR-21 plasmids influence PTEN protein expression levels in PC-9 cells, assessed by western blot analysis. (B) Statistical analysis of PTEN protein expression levels in vitro. ${ }^{* *} \mathrm{P}<0.01 \mathrm{vs.} 0 \mathrm{nM}$ triptolide-treated group; ${ }^{\# \#} \mathrm{P}<0.01$ vs. $25 \mathrm{nM}$ triptolide-treated group. miR-21, microRNA 21; PTEN, phosphatase and tensin homolog.

blotting. As demonstrated in Fig. 6, pre-treatment with triptolide $(25$ or $50 \mathrm{nM})$ enhanced PTEN protein expression levels in PC-9 cell compared with the control group $(\mathrm{P}<0.01)$. These data suggested that triptolide may increase PTEN protein expression levels in human NSCLC cells.

Upregulation of miR-21 influences the effect of triptolide on cell viability of PC-9 cells. In order to further study the effects of triptolide on cell viability, miR-21 and negative control plasmids were transfected into PC-9 cells with Lipofectamine 2000 reagent. As demonstrated in Fig. 7A, RT-qPCR analysis indicated that miR-21 plasmids promoted miR-21 expression in the PC-9 cells, following 2-day triptolide $(25 \mathrm{nM})$ treatment, compared with the triptolide-treated group $(\mathrm{P}<0.01)$. The reduction of cell viability following triptolide treatment $(\mathrm{P}<0.01$ vs. untreated control) was partially reversed by miR-21 plasmid transfection. The miR-21 plasmid-transfected group exhibited significantly increased cell viability compared with the triptolide-treated group $(\mathrm{P}<0.01$; Fig. 7B).These data suggested that triptolide reduces proliferation in human NSCLC cells via downregulation of miR-21.

Upregulation of miR-21 influences the effect of triptolide on PTEN protein expression in PC-9 cells. In order to decipher the effect of triptolide on the cell viability of PC-9 cells, miR-21 and negative control plasmids were transfected into PC-9 cells. As demonstrated in Fig. 8, miR-21 plasmid transfection suppressed PTEN protein expression levels in PC-9 cells, compared with the $25 \mathrm{nM}$ triptolide-treated group $(\mathrm{P}<0.01)$. These data that suggested triptolide reduces proliferation in human NSCLC cells via the upregulation of PTEN expression levels.

\section{Discussion}

Lung carcinoma is the type of malignant tumor with the highest rate of mortality worldwide with 1.2 million diagnoses per year of various types of which $80 \%$ are NSCLC (15). At the point 
of diagnosis, $65-70 \%$ of cases of NSCLC are in the late phase of the disease. Due to this, surgery is no longer a viable option as treatment, and chemotherapy becomes the key treatment strategy (16). At present, the most effective drug for NSCLC is platinum and the three generations of novel platinum-based drugs introduced from 1990 onwards (17). The chemotherapy efficiency of the late phase of NSCLC is $20-40 \%$, with the median survival period being $<10$ months (18). The results of the present study indicated that the administration of triptolide effectively attenuated cell viability, induced cell apoptosis and activated caspase- 3 and 9 in PC-9 cells. Previous studies have demonstrated that triptolide induced cell apoptosis in gastric (19), breast (20) and cholangiocarcinoma (21) cancer. The data from those studies and the present study, suggest a potential antitumor effect of triptolide on human NSCLC cells. However, this requires further investigation.

miRs are a newly identified type of non-coding small RNA, which exist in eukaryotes (22). miR molecules regulate targeted gene expression through specific-protein mRNA degradation or inhibition of translation, and are involved in cell growth, differentiation, apoptosis, and other important cell processes $(22,23)$. miR-21 is one of the earliest discovered miRNA molecules, and its gene is located in the 3'UTR of the vacuole membrane protein 1 gene at chromosome $17 q 23.1$ (24). This region is usually amplified in neuroblastomas and colon, breast and lung cancer (24). This is consistent with miR-21 overexpression in various types of cancer (25). Yang et al (26) indicated that the downregulation of miR-21 expression suppresses NSCLC cell proliferation through the upregulation of programmed cell death. Data from the current study also suggested that triptolide reduced miR-21 expression in PC-9 cells. Li et al (27) indicated that triptolide inhibits miR-21 expression levels, enhances PTEN expression levels and modulates the sensitivity of K562/A02 cells.

In the field of cancer research, researchers have identified various PTEN gene mutations and deletions, that lead to the formation of a defective protein, and abnormal protein expression (28). Studying the role of PTEN in tumor malignancies may contribute to an understanding of cellular signal transduction mechanisms, and open up a novel direction for genetic therapies (29). Studies have observed that PTEN is abnormally expressed in various types of cancer (30). Research on PTEN and NSCLC association have been reported (31) and in the present study, data demonstrated that triptolide enhanced PTEN protein expression levels in PC-9 cells compared with the control group. However, upregulation of miR-21 expression levels suppressed the effect of triptolide on cell viability and PTEN protein expression levels in PC-9 cells.

In conclusion, the results of the present study demonstrated that triptolide reduces the proliferation and enhances the apoptosis of human NSCLC cells by targeting miR-21 via PTEN. The current study enhances the understanding of triptolide treatment and the regulatory mechanisms of miR-21 in cancer progression.

\section{Acknowledgements}

This study was supported by the Hebei University Special Funds for Medical Science Construction project (grant no. 2015B1001).

\section{References}

1. Pu Z, Yuan X, Zhang X, Chen Q and Xie H: Meta-analysis on the association between CYP2D6*10 gene polymorphism and disease free survival of breast cancer patients receiving tamoxifen treatment in Asia. Bangladesh J Pharmacol 9: 652-662, 2014.

2. Couto P, Miranda D, Vieira R, Vilhena A, De Marco L and Bastos-Rodrigues L: Association between CLOCK, PER3 and CCRN4L with non-small cell lung cancer in Brazilian patients. Mol Med Rep 10: 435-440, 2014.

3. Zhang HM, Yang FQ, Yan Y, Che JP and Zheng JH: High expression of long non-coding RNA SPRY4-IT1 predicts poor prognosis of clear cell renal cell carcinoma. Int J Clin Exp Pathol 7: 5801-5809, 2014.

4. Faltejskova P, Besse A, Sevcikova S, Kubiczkova L, Svoboda M, Smarda J, Kiss I, Vyzula R and Slaby O: Clinical correlations of miR-21 expression in colorectal cancer patients and effects of its inhibition on DLD1 colon cancer cells. Int J Colorectal Dis 27: 1401-1408, 2012.

5. Zheng J, Xue H, Wang T, Jiang Y, Liu B, Li J, Liu Y, Wang W, Zhang B and Sun M: miR-21 downregulates the tumor suppressor P12 CDK2AP1 and stimulates cell proliferation and invasion. J Cell Biochem 112: 872-880, 2011.

6. Hu Y, Wang C, Li Y, Zhao J, Chen C, Zhou Y, Tao Y, Guo M, Qin N, Ren T, et al: MiR-21 controls in situ expansion of CCR6 ${ }^{+}$ regulatory $\mathrm{T}$ cells through PTEN/AKT pathway in breast cancer. Immunol Cell Biol 93: 753-764, 2015.

7. Zhu Y, Yu X, Fu H, Wang H, Wang P, Zheng X and Wang Y: MicroRNA-21 is involved in ionizing radiation-promoted liver carcinogenesis. Int J Clin Exp Med 3: 211-222, 2010

8. Nassar FJ, El Sabban M, Zgheib NK, Tfayli A, Boulos F, Jabbour M, El Saghir NS, Talhouk R, Bazarbachi A, Calin GA, et al: miRNA as potential biomarkers of breast cancer in the Lebanese population and in young women: A pilot study. PLoS One 9: e107566, 2014.

9. Jiang Y, Chen X, Tian W, Yin X, Wang J and Yang H: The role of TGF-ß1-miR-21-ROS pathway in bystander responses induced by irradiated non-small-cell lung cancer cells. Br J Cancer 111: 772-780, 2014.

10. Kobayashi H, Ohno S, Sasaki Y and Matsuura M: Hereditary breast and ovarian cancer susceptibility genes (review). Oncol Rep 30: 1019-1029, 2013.

11. Nishimura R, Arima N, Toyoshima S, Ohi Y, Anan K, Sagara Y, Mitsuyama S and Tamura K: Evaluation of PTEN loss and PIK3CA mutations and their correlation with efficacy of trastuzumab treatment in HER2-positive metastatic breast cancer: A retrospective study (KBC-SG 1001). Mol Clin Oncol 1: 47-52, 2013.

12. Jiang N, Dong XP, Zhang SL, You QY, Jiang XT and Zhao XG: Triptolide reverses the Taxol resistance of lung adenocarcinoma by inhibiting the NF-kappaB signaling pathway and the expression of NF-kappaB-regulated drug-resistant genes. Mol Med Rep: Nov 2, 2015 (Epub ahead of print).

13. Zhou H, Guo W, Long $C$, Wang $H$, Wang $J$ and Sun X: Triptolide inhibits proliferation of Epstein-Barr virus-positive B lymphocytes by down-regulating expression of a viral protein LMP1. Biochem Biophys Res Commun 456: 815-820, 2015.

14. Ho JN, Byun SS, Lee S, Oh JJ, Hong SK, Lee SE and Yeon JS: Synergistic antitumor effect of triptolide and cisplatin in cisplatin resistant human bladder cancer cells. J Urol 193: 1016-1022, 2015.

15. Kriegshäuser G, Fabjani G,Ziegler B,Zöchbauer-Müller S, End A and Zeillinger R: Biochip-based detection of KRAS mutation in non-small cell lung cancer. Int J Mol Sci 12: 8530-8538, 2011.

16. Shames DS, Girard L, Gao B, Sato M, Lewis CM, Shivapurkar N, Jiang A, Perou CM, Kim YH, Pollack JR, et al: A genome-wide screen for promoter methylation in lung cancer identifies novel methylation markers for multiple malignancies. PLoS Med 3: e486, 2006.

17. Bognar CL, Simon SD, Gansl RC, Abramoff R, Aisen M, Lopes Junior Gde L, Smaletz O, Peres SV and Tabacof J: The impact of erlotinib use in non-small-cell lung cancer patients treated in a private reference general hospital and in a private cancer clinic from 2005 to 2011. Einstein (Sao Paulo) 13: 215-220, 2015 (In English, Portuguese).

18. Moravcikova E, Krepela E, Prochazka J, Benkova K and Pauk N: Differential sensitivity to apoptosome apparatus activation in non-small cell lung carcinoma and the lung. Int J Oncol 44: 1443-1454, 2014. 
19. Wang BY, Cao J, Chen JW and Liu QY: Triptolide induces apoptosis of gastric cancer cells via inhibiting the overexpression of MDM2. Med Oncol 31: 270, 2014.

20. Shao H, Ma J, Guo T and Hu R: Triptolide induces apoptosis of breast cancer cells via a mechanism associated with the Wnt/ß-catenin signaling pathway. Exp Ther Med 8: 505-508, 2014

21. Ding X, Zhang B, Pei Q, Pan J, Huang S, Yang Y, Zhu Z, Lv Y and Zou X: Triptolide induces apoptotic cell death of human cholangiocarcinoma cells through inhibition of myeloid cell leukemia-1. BMC Cancer 14: 271, 2014.

22. Rothschild SI: microRNA therapies in cancer. Mol Cell Ther 2: 7, 2014

23. Li JS and Yao ZX: MicroRNAs: novel regulators of oligodendrocyte differentiation and potential therapeutic targets in demyelination-related diseases. Mol Neurobiol 45: 200-212, 2012.

24. Pan X, Wang ZX and Wang R: MicroRNA-21: A novel therapeutic target in human cancer. Cancer Biol Ther 10: 1224-1232, 2010.

25. Gong B, Liu WW, Nie WJ, Li DF, Xie ZJ, Liu C, Liu YH, Mei P and Li ZJ: MiR-21/RASA1 axis affects malignancy of colon cancer cells via RAS pathways. World J Gastroenterol 21: 1488-1497, 2015.
26. Yang Y, Meng H, Peng Q, Yang X, Gan R, Zhao L, Chen Z, Lu J and Meng QH: Downregulation of microRNA-21 expression restrains non-small cell lung cancer cell proliferation and migration through upregulation of programmed cell death 4 Cancer Gene Ther 22: 23-29, 2015.

27. Li H, Hui L, Xu W, Shen H, Chen Q, Long L and Zhu X: Triptolide modulates the sensitivity of K562/A02 cells to adriamycin by regulating miR-21 expression. Pharm Biol 50: 1233-1240, 2012.

28. Bunney TD and Katan M: Phosphoinositide signalling in cancer: Beyond PI3K and PTEN. Nat Rev Cancer 10: 342-352, 2010.

29. Zhang JG, Wang JJ, Zhao F, Liu Q, Jiang K and Yang GH MicroRNA-21 (miR-21) represses tumor suppressor PTEN and promotes growth and invasion in non-small cell lung cancer (NSCLC). Clin Chim Acta 411: 846-852, 2010.

30. Tian T, Nan KJ, Guo H, Wang WJ, Ruan ZP, Wang SH, Liang X and Lu CX: PTEN inhibits the migration and invasion of HepG2 cells by coordinately decreasing MMP expression via the PI3K/Akt pathway. Oncol Rep 23: 1593-1600, 2010.

31. Poliseno L and Pandolfi PP: PTEN ceRNA networks in human cancer. Methods 77-78: 41-50, 2015. 